TAMKANG JOURNAL OF MATHEMATICS

Volume 33, Number 3, Autumn 2002

\title{
INEQUALITIES FOR TERMINAL VALUE PROBLEMS FOR DIFFERENTIAL EQUATIONS
}

\author{
S. B. PACHPATTE AND B. G. PACHPATTE
}

\begin{abstract}
The aim of the present paper is to establish some new integral inequalities which provide explicit bounds on unknown functions. The inequalities given here can be used as handy tools in the study of terminal value problems for certain differential equations.
\end{abstract}

\section{Introduction}

The classical integral inequalities which give explicit bounds for an unknown function have played a foundamental role in establishing the foundations of the theory of differential and integral equations. Integral inequalities of the Gronwall-Bellman type are frequently used in various contexts. Over the years several such inequalities have been developed and used considerably to study the various problems in the theory of differential and integral equations, see $[1,2]$ and the references therein. In the present paper, we offer some fundamental integral inequalities, which can be used as tools in the analysis of terminal value problems for certain differental equations.

\section{Main Results}

In what follows, we donote by $R$ the set of real numbers and $R_{+}=[0, \infty)$. We assume that all the integrals involved throughout the discussion exist on the respective domains of their definitions.

An interesting and useful integral inequality is established in the following theorem.

Theorem 1. Let $u(t), a(t), b(t)$ be real-valued nonnegative continuous functions defined for $t \in R_{+}$and suppose that $a(t)$ is nonincreasing for $t \in R_{+}$. If

$$
u(t) \leq a(t)+\int_{t}^{\infty} b(s) u(s) d s
$$

Received October 12, 2000.

2000 Mathematics Subject Classification. Primary 26D10, 26D15.

Key words and phrases. Inequalities, terminal value problems, differential equations, explicit bounds, Gronwall-Bellman type, two independendent variable generalization, dependency of solutions. 
for $t \in R_{+}$, then

$$
u(t) \leq a(t) \exp \left(\int_{t}^{\infty} b(s) d s\right)
$$

for $t \in R_{+}$.

Proof. First we assume that $a(t)>0$ for $t \in R_{+}$. From (2.1) it is easy to observe that

$$
\frac{u(t)}{a(t)} \leq 1+\int_{t}^{\infty} b(s) \frac{u(s)}{a(s)} d s
$$

Define a function $z(t)$ by the right side of $(2.3)$, then $z(\infty)=1, \frac{u(t)}{a(t)} \leq z(t)$ and

$$
z^{\prime}(t)=-b(t) \frac{u(t)}{a(t)} \geq-b(t) z(t)
$$

The inequality (2.4) implies the estimate

$$
z(t) \leq \exp \left(\int_{t}^{\infty} b(s) d s\right)
$$

Using (2.5) in $\frac{u(t)}{a(t)} \leq z(t)$, we get the desired inequality in (2.2).

If $a(t)$ is nonnegative, we carry out the above procedure with $a(t)+\epsilon$ instead of $a(t)$, where $\epsilon>0$ is an arbitrary small constant, and subsequently pass to the limit as $\epsilon \rightarrow 0$ to obtain $(2.2)$.

We next establish the following inequality which can be used in more general situations.

Theorem 2. Let $u(t), a(t), b(t)$, be as in Theorem 1 and $L: R_{+}^{2} \rightarrow R_{+}$be a continuous function which satisfies the condition

$$
0 \leq L(t, u)-L(t, v) \leq M(t, v)(u-v)
$$

for $u \geq v \geq 0$, where $M(t, v)$ is a real-valued nonnegative continuous function defined for $t, v \in R_{+}$. If

$$
u(t) \leq a(t)+\int_{t}^{\infty} b(s) u(s) d s+\int_{t}^{\infty} L(s, u(s)) d s,
$$

for $t \in R_{+}$, then

$$
u(t) \leq E(t)\left[a(t)+A(t) \exp \left(\int_{t}^{\infty} M(s, E(s) a(s)) E(s) d s\right)\right],
$$


for $t \in R_{+}$, where

$$
\begin{aligned}
& E(t)=\exp \left(\int_{t}^{\infty} b(s) d s\right), \\
& A(t)=\int_{t}^{\infty} L(s, E(s) a(s)) d s
\end{aligned}
$$

for $t \in R_{+}$.

Proof. Define a function $z(t)$ by

$$
z(t)=\int_{t}^{\infty} L(s, u(s)) d s
$$

then (2.6) can be restated as

$$
u(t) \leq a(t)+z(t)+\int_{t}^{\infty} b(s) u(s) d s .
$$

Since $a(t)+z(t)$ is nonnegative, continuous and nonincreasing for $t \in R_{+}$, by applying Theorem 1 to (2.11) we have

$$
u(t) \leq(a(t)+z(t)) E(t) .
$$

From (2.10) and (2.12) and the hypotheses on $L$, we observe that

$$
\begin{aligned}
z(t) & \leq \int_{t}^{\infty}[L(s, E(s) a(s)+E(s) z(s))-L(s, E(s) a(s))+L(s, E(s) a(s))] d s \\
& \leq A(t)+\int_{t}^{\infty} M(s, E(s) a(s)) E(s) z(s) d s .
\end{aligned}
$$

Clearly, $A(t)$ is nonnegative, continuous and nonincreasing for $t \in R_{+}$. Now an application of Theorem 1 to (2.13) yields

$$
z(t) \leq A(t) \exp \left(\int_{t}^{\infty} M(s, E(s) a(s)) E(s) d s\right) .
$$

Using (2.14) in (2.12) we get the required inequality in (2.7).

The inequalities established in the following theorems can be used in certain applications.

Theorem 3. Let $u(t), a(t), b(t)$ be real-valued nonnegative continuous functions defined for $t \in R_{+}$and $L, M$ be as in Theorem 2 . If

$$
u(t) \leq a(t)+b(t) \int_{t}^{\infty} L(s, u(s)) d s
$$


for $t \in R_{+}$, then

$$
u(t) \leq a(t)+b(t) e(t) \exp \left(\int_{t}^{\infty} M(s, a(s)) b(s) d s\right)
$$

for $t \in R_{+}$, where

$$
e(t)=\int_{t}^{\infty} L(s, a(s)) d s
$$

for $t \in R_{+}$.

Proof. Define a function $z(t)$ by (2.10). Then from (2.15), we have

$$
u(t) \leq a(t)+b(t) z(t) .
$$

From (2.10), (2.18) and the hypotheses on $L$, we observe that

$$
\begin{aligned}
z(t) & \leq \int_{t}^{\infty}[L(s, a(s)+b(s) z(s))-L(s, a(s))+L(s, a(s))] d s \\
& \leq e(t)+\int_{t}^{\infty} M(s, a(s)) b(s) z(s) d s,
\end{aligned}
$$

where $e(t)$ is defined by (2.17). Clearly $e(t)$ is real-valued nonnegative, continuous and nonincreasing in $t \in R_{+}$. An application of Theorem 1 to (2.19) yields

$$
z(t) \leq e(t) \exp \left(\int_{t}^{\infty} M(s, a(s)) b(s) d s\right) .
$$

The desired inequality in (2.16) follows from (2.18) and (2.20).

Theorem 4. Let $u(t), a(t), b(t)$ be as in Theorem 3 and $L: R_{+}^{2} \rightarrow R_{+}$be a continuous function which satisfies the condition

$$
0 \leq L(t, u)-L(t, v) \leq M(t, v) \phi^{-1}(u-v),
$$

for $u \geq v \geq 0$, where $M(t, v)$ is defined as in Theorem $2, \phi: R_{+} \rightarrow R_{+}$be a continuous and strictly increasing function with $\phi(0)=0, \phi^{-1}$ is the inverse function of $\phi$ and

$$
\phi^{-1}(u v) \leq \phi^{-1}(u) \phi^{-1}(v),
$$

for $u, v \in R_{+}$. If

$$
u(t) \leq a(t)+b(t) \phi\left(\int_{t}^{\infty} L(s, u(s)) d s\right)
$$

for $t \in R_{+}$, then

$$
u(t) \leq a(t)+b(t) \phi\left(e(t) \exp \left(\int_{t}^{\infty} M(s, a(s)) \phi^{-1}(b(s)) d s\right)\right),
$$


for $t \in R_{+}$, where $e(t)$ is defined by (2.17).

Proof. Define a function $z(t)$ by (2.10), then from (2.21) we have

$$
u(t) \leq a(t)+b(t) \phi(z(t)) .
$$

From (2.10), (2.23) and the hypotheses on $L$ and $\phi$, it is easy to observe that

$$
\begin{aligned}
z(t) & \leq \int_{t}^{\infty}[L(s, a(s)+b(s) \phi(z(s)))-L(s, a(s))+L(s, a(s))] d s \\
& \leq e(t)+\int_{t}^{\infty} M(s, a(s)) \phi^{-1}(b(s)) \phi(z(s)) d s \\
& \leq e(t)+\int_{t}^{\infty} M(s, a(s)) \phi^{-1}(b(s)) z(s) d s,
\end{aligned}
$$

where $e(t)$ is defined by (2.17). Now by following the last arguments as in the proof of Theorem 3 , we get the required inequality in (2.22).

\section{Two Independent Variable Generalizations}

In this section, we establish two independent variable versions of Theorems 1-4, which can be used as tools in the study of terminal value problems for certain hyperbolic partial differential equations.

The following result is the two independent variable version of the inequality given in Theorem 1.

Theorem 5. Let $u(x, y), a(x, y) . b(x, y)$ be real-valued nonnegative continuous functions defined for $x, y \in R_{+}$and suppose that $a(x, y)$ is nonincreasing in $x, y \in R_{+}$. If

$$
u(x, y) \leq a(x, y)+\int_{x}^{\infty} \int_{y}^{\infty} b(s, t) u(s, t) d t d s,
$$

for $x, y \in R_{+}$, then

$$
u(x, y) \leq a(x, y) \exp \left(\int_{x}^{\infty} \int_{y}^{\infty} b(s, t) d t d s\right)
$$

for $x, y \in R_{+}$.

Proof. First we assume that $a(x, y)>0$ for $x, y \in R_{+}$. From (3.1) it is easy to observe that

$$
\frac{u(x, y)}{a(x, y)} \leq 1+\int_{x}^{\infty} \int_{y}^{\infty} b(s, t) \frac{u(s, t)}{a(s, t)} d t d s .
$$

Define a function $z(x, y)$ by the right side of (3.3). Then $z(x, \infty)=z(\infty, y)=1$, 
$\frac{u(x, y)}{a(x, y)} \leq z(x, y)$ and $z_{x y}(x, y)=b(x, y) \frac{u(x, y)}{a(x, y)} \leq b(x, y) z(x, y)$.

The rest of the proof can be completed by following the proof of Theorem 4.2.1 give in [2, p. 324] with suitable changes and closely looking at the proof of Theorem 1 given above.

We next establish the two independent variable versions of the Theorems $2-4$, whose proofs follows by colosely looking at the proofs of Theorems 2-4, and making use of Theorem 5 .

Theorem 6. Let $u(x, y), a(x, y), b(x, y)$ be as in Theorem 5 and $L: R_{+}^{3} \rightarrow R_{+}$be a continuous function which satisfies the condition

$$
0 \leq L(x, y, u)-L(x, y, v) \leq M(x, y, v)(u-v)
$$

for $u \geq v \geq 0$, where $M(x, y, v)$ is a real-valued nonnegative continuous function defined for $x, y, v \in R_{+}$. If

$$
u(x, y) \leq a(x, y)+\int_{x}^{\infty} \int_{y}^{\infty} b(s, t) u(s, t) d t d s+\int_{x}^{\infty} \int_{y}^{\infty} L(s, t, u(s, t)) d t d s
$$

for $x, y \in R_{+}$, then

$$
u(x, y) \leq F(x, y)\left[a(x, y)+B(x, y) \exp \left(\int_{x}^{\infty} \int_{y}^{\infty} M(s, t, F(s, t) a(s, t))\right) F(s, t) d t d s\right],
$$

for $x, y \in R_{+}$, where

$$
\begin{aligned}
& F(x, y)=\exp \left(\int_{x}^{\infty} \int_{y}^{\infty} b(s, t) d t d s\right) \\
& B(x, y)=\int_{x}^{\infty} \int_{y}^{\infty} L(s, t, F(s, t) a(s, t)) d t d s
\end{aligned}
$$

for $x, y \in R_{+}$

Theorem 7. Let $u(x, y), a(x, y), b(x, y)$ be real-valued, nonnegative and continuous functions defined for $x, y \in R_{+}$and $L, M$ be as in Theorem 6 . If

$$
u(x, y) \leq a(x, y)+b(x, y) \int_{x}^{\infty} \int_{y}^{\infty} L(s, t, u(s, t)) d t d s,
$$

for $x, y \in R_{+}$, then

$$
u(x, y) \leq a(x, y)+b(x, y) f(x, y) \exp \left(\int_{x}^{\infty} \int_{y}^{\infty} M(s, t, a(s, t)) b(s, t) d t d s\right)
$$


for $x, y \in R_{+}$, where

$$
f(x, y)=\int_{x}^{\infty} \int_{y}^{\infty} L(s, t, a(s, t)) d t d s
$$

for $x, y \in R_{+}$.

Theorem 8. Let $u(x, y), a(x, y), b(x, y)$ be as in Theorem 7 and $L: R_{+}^{3} \rightarrow R_{+}$be a continuous function wich satisfies the condition

$$
0 \leq L(x, y, u)-L(x, y, v) \leq M(x, y, v) \psi^{-1}(u-v),
$$

for $u \geq v \geq 0$, where $M(x, y, v)$ is as defined in Theorem $6, \psi: R_{+} \rightarrow R_{+}$is a continuous and strictly increasing function with $\psi(0)=0, \psi^{-1}$ is the inverse function of $\psi$ and $\psi^{-1}(u v) \leq \psi^{-1}(u) \psi^{-1}(v)$, for $u, v \in R_{+}$. If

$$
u(x, y) \leq a(x, y)+b(x, y) \psi\left(\int_{x}^{\infty} \int_{y}^{\infty} L(s, t, u(x, y)) d t d s\right)
$$

for $x, y \in R_{+}$, then

$$
u(x, y) \leq a(x, y)+b(x, y) \psi\left(f(x, y) \exp \left(\int_{x}^{\infty} \int_{y}^{\infty} M(s, t, a(s, t)) \psi^{-1}(b(s, t))\right) d t d s\right),
$$

for $x, y \in R_{+}$, where $f(x, y)$ is defined by (3.10).

\section{Applications to Terminal Value Problems}

In this section, we present some immediate applications of Theorem 1 to study certain properties of solutions of the following terminal value problem for differential equation

$$
\begin{aligned}
u^{\prime}(t) & =f(t, u(t))+p(t), \\
u(\infty) & =u_{\infty},
\end{aligned}
$$

where $f: R_{+} \times R \rightarrow R, p: R_{+} \rightarrow R$ are continuous functions and $u_{\infty} \in R$.

The following theorem deals with the estimate on the solution of problem $(P)-\left(P_{\infty}\right)$.

Theorem 9. Suppose that

$$
\begin{aligned}
|f(t, u)| & \leq b(t)|u|, \\
\left|u_{\infty}-Q(t)\right| & \leq a(t),
\end{aligned}
$$

where $a(t), b(t)$ are as defined in Theorem 1 and $Q(t)=\int_{t}^{\infty} p(s) d s$.

If $u(t)$ is a solution of $(P)-\left(P_{\infty}\right)$, then

$$
|u(t)| \leq a(t) \exp \left(\int_{t}^{\infty} b(s) d s\right),
$$


for $t \in R_{+}$.

Proof. If $u(t)$ is a solution of $(P)-\left(P_{\infty}\right)$, then it can be written as (see $[1$, p. 80])

$$
u(t)=u_{\infty}-\int_{t}^{\infty}[f(s, u(s))+p(s)] d s
$$

for $t \in R_{+}$. From (4.4), (4.1), (4.2), we observe that

$$
|u(t)| \leq a(t)+\int_{t}^{\infty} b(s)|u(s)| d s .
$$

Now an application of Theorem 1 to (4.5) yields the required estimate in (4.3).

Next we shall prove the uniqueness of the solutions of problem $(P)-\left(P_{\infty}\right)$.

Theorem 10. Suppose that the function $f$ in $(P)$ satisfies the condition

$$
|f(t, u)-f(t, v)| \leq b(t)|u-v|
$$

where $b(t)$ is defined as in Theorem 1 . Then the problem $(P)-\left(P_{\infty}\right)$ has at most one solution on $R_{+}$.

Proof. The problem $(P)-\left(P_{\infty}\right)$ is equivalent to the integral equation (4.4). Let $u(t)$ and $v(t)$ be two solutions of $(P)-\left(P_{\infty}\right)$ on $R_{+}$. From (4.4) and (4.6) we have

$$
|u(t)-v(t)| \leq \int_{t}^{\infty} b(s)|u(s)-v(s)| d s .
$$

Now an application of Theorem 1 to (4.7) yields $u(t)=v(t)$ i.e. there is at most one solution of the problem $(P)-\left(P_{\infty}\right)$.

Our next result shows the dependency of solutions of $(P)-\left(P_{\infty}\right)$ on terminal values.

Theorem 11. Let $u_{1}(t)$ and $u_{2}(t)$ be the solutions of $(P)$ with the given terminal conditions

$$
u_{1}(\infty)=u_{1 \infty}
$$

and

$$
u_{2}(\infty)=u_{2 \infty}, \quad\left(P_{2 \infty}\right)
$$

respectively, where $u_{1 \infty}, u_{2 \infty} \in R$. Suppose that the function $f$ in $(P)$ satisfies the condition (4.6) in Theorem 10. Then

$$
\left|u_{1}(t)-u_{2}(t)\right| \leq\left|u_{1 \infty}-u_{2 \infty}\right| \exp \left(\int_{t}^{\infty} b(s) d s\right),
$$

for $t \in R_{+}$. 
Proof. By using the facts that $u_{1}(t)$ and $u_{2}(t)$ are the solutions of $(P)-\left(P_{1 \infty}\right)$ and $(P)-\left(P_{2} \infty\right)$ respectively, we have

$$
u_{1}(t)-u_{2}(t)=u_{1 \infty}-u_{2 \infty}-\int_{t}^{\infty}\left[f\left(s, u_{1}(s)\right)-f\left(s, u_{2}(s)\right)\right] d s .
$$

From (4.9), (4.6), we have

$$
\left|u_{1}(t)-u_{2}(t)\right| \leq\left|u_{1 \infty}-u_{2 \infty}\right|+\int_{t}^{\infty} b(s)\left|u_{1}(s)-u_{2}(s)\right| d s .
$$

Now an application of Theorem 1 to (4.10) yields the required estimate in (4.8).

We next consider the following differential equations

$$
\begin{aligned}
& u^{\prime}(t)=f(t, u(t), \mu), \\
& u^{\prime}(t)=f\left(t, u(t), \mu_{0}\right),
\end{aligned}
$$

with the given terminal value condition $\left(\mathrm{P}_{\infty}\right)$, where $f: R_{+} \times R^{2} \rightarrow R$ is a continuous function and $\mu, \mu_{0}$ are real parameters.

The following theorem shows the dependency of soutions of $(4.11)-\left(P_{\infty}\right)$ and (4.12)$\left(P_{\infty}\right)$ on pure paremters.

Theorem 12. Suppose that the function $f$ satisfies the conditions

$$
\begin{aligned}
|f(t, u, \mu)-f(t, v, \mu)| & \leq b(t)|u-v|, \\
\left|f(t, u, \mu)-f\left(t, u, \mu_{0}\right)\right| & \leq c(t)\left|\mu-\mu_{0}\right|,
\end{aligned}
$$

where $b(t), c(t): R_{+} \rightarrow R_{+}$are continuous functions. If $u_{1}(t)$ and $u_{2}(t)$ are the solutions of (4.11)- $\left(\mathrm{P}_{\infty}\right)$ and $(4.12)-\left(P_{\infty}\right)$ for $t \in R_{+}$respectively, then

$$
\left|u_{1}(t)-u_{2}(t)\right| \leq B(t) \exp \left(\int_{t}^{\infty} b(s) d s\right)
$$

for $t \in R_{+}$, where

$$
B(t)=\left|\mu-\mu_{0}\right| \int_{t}^{\infty} c(s) d s,
$$

for $t \in R_{+}$.

Proof. Let $z(t)=u_{1}(t)-u_{2}(t), t \in R_{+}$. As in the proof of Theorem 11, from the hypotheses, we observe that

$$
\begin{aligned}
z(t) & =\int_{t}^{\infty}\left[f\left(s, u_{1}(s), \mu\right)-f\left(s, u_{2}(s), \mu\right)+f\left(s, u_{2}(s), \mu\right)-f\left(s, u_{2}(s), \mu_{0}\right)\right] d s \\
& \leq B(t)+\int_{t}^{\infty} b(s)|z(s)| d s
\end{aligned}
$$


where $B(t)$ is defined by (4.16). Clearly $B(t)$ is nonnegative, continuous and nonincreasing for $t \in R_{+}$. Now an application of Theorem 1 to (4.17) yields the required estimate in (4.15).

In concluding, we note that the inequality given in Theorem 5 can be used to study the similar properties as in Theorems 9-12 for the terminal value problem for hyperbolic partial differential equation of the form

$$
\begin{gathered}
u_{x y}(x, y)=f(x, y, u(x, y))+p(x, y), \\
u(x, \infty)=\sigma_{\infty}(x), u(\infty, y)=\tau_{\infty}(y), u(\infty, \infty)=k,
\end{gathered}
$$

where $f: R_{+}^{2} \times R \rightarrow R, \sigma_{\infty}, \tau_{\infty}: R_{+} \rightarrow R$ are continuous functions and $k$ is a real constant, under some suitable conditions on the functions involved in $(\mathrm{H})-\left(\mathrm{H}_{\infty}\right)$ and the constank $k$. Since the details of these results are very close to those given above, we omit it here. Various applications of the other inequalities will be reported elsewhere.

\section{References}

[1] D. Bainov and P. Simeonov, Integral Inequalities and Applications, Kluwer Academic Publishers Dordrecht, 1992.

[2] B. G. Pachpatte, Inequalities for Differential and Integral Equations, Academic Press, New York and London, 1998.

57, Shri Niketan Colony, Near Abhinay Talkies, Aurangabad 431 001, (Maharashtra) India. 